\title{
The Multi-site All-Sky CAmeRA (MASCARA)
}

\section{Finding transiting exoplanets around bright $\left(m_{\mathrm{V}}<8\right)$ stars}

\author{
G. J. J. Talens ${ }^{1}$, J. F. P. Spronck ${ }^{1}$, A.-L. Lesage ${ }^{1}$, G. P. P. L. Otten ${ }^{1}$, R. Stuik ${ }^{1}$, D. Pollacco ${ }^{2}$, and I. A. G. Snellen ${ }^{1}$ \\ 1 Leiden Observatory, Leiden University, Postbus 9513, 2300 RA Leiden, The Netherlands \\ e-mail: talens@strw.leidenuniv.nl \\ 2 Department of Physics, University of Warwick, Coventry CV4 7AL, UK
}

Received 22 December 2016 / Accepted 9 February 2017

\begin{abstract}
This paper describes the design, operations, and performance of the Multi-site All-Sky CAmeRA (MASCARA). Its primary goal is to find new exoplanets transiting bright stars, $4<m_{V}<8$, by monitoring the full sky. MASCARA consists of one northern station on La Palma, Canary Islands (fully operational since February 2015), one southern station at La Silla Observatory, Chile (operational from early 2017), and a data centre at Leiden Observatory in the Netherlands. Both MASCARA stations are equipped with five interline CCD cameras using wide field lenses ( $24 \mathrm{~mm}$ focal length) with fixed pointings, which together provide coverage down to airmass 3 of the local sky. The interline CCD cameras allow for back-to-back exposures, taken at fixed sidereal times with exposure times of 6.4 sidereal seconds. The exposures are short enough that the motion of stars across the CCD does not exceed one pixel during an integration. Astrometry and photometry are performed on-site, after which the resulting light curves are transferred to Leiden for further analysis. The final MASCARA archive will contain light curves for $\sim 70000$ stars down to $m_{V}=8.4$, with a precision of $1.5 \%$ per 5 minutes at $m_{V}=8$.
\end{abstract}

Key words. surveys - planetary systems - eclipses - telescopes - instrumentation: detectors

\section{Introduction}

When the first hot Jupiters were discovered twenty years ago (Mayor \& Queloz 1995; Butler et al. 1997), it was realized that these sorts of planets have a significant probability to exhibit transits. The detection of these transits would confirm the hypothesis that the observed stellar radial velocity variations were indeed caused by the gravitational interaction with planets, and furthermore allow the determination of their size and true mass. Hence, all exoplanet systems newly discovered using the radial velocity method were photometrically monitored to search for transit signatures. The first transiting planet found in this way was HD 209458b (Henry et al. 2000; Charbonneau et al. 2000) and the subsequent detection of an atmospheric signature (Charbonneau et al. 2002) further strengthened the scientific value of transiting exoplanets. HD 209458b is still one of the two most-studied hot Jupiter systems known to date, together with HD 189733b (Bouchy et al. 2005) which orbits a similarly bright star.

Initial attempts to directly search for transit signals via high-precision photometric monitoring of large star fields, such as Vulcan (Borucki et al. 2001) and STARE (Brown \& Charbonneau 2000), failed to find planets. This was possibly due to an underestimation of the amount of observing time required (Horne 2001). It was not until the discovery of OGLE-TR-56 by the OGLE-III team (Konacki et al. 2003) that transit surveys proved to be a viable way of finding exoplanets. However, the OGLE-III targets were generally faint, $m_{V}>15$, making radial velocity follow-up challenging. Subsequent transit surveys that targeted brighter stars have found many planets, starting with TrES (using three $10 \mathrm{~cm}$ telescopes; Alonso et al. 2004) and XO (using two lenses with $200 \mathrm{~mm}$ focal length; McCullough et al. 2005) which found five and six transiting planets, respectively, in the $m_{V}=10-13$ magnitude range. The larger SuperWASP (Pollacco et al. 2006) and HATNet surveys (Bakos et al. 2004) have now been operational for more than a decade, each using a battery of wide-field lenses and finding a total of about two hundred planets orbiting stars in the $m_{V}=10-13$ magnitude range. In addition, they found a few that are significantly brighter, for example WASP-33 $\left(m_{V}=8.3\right.$, Collier Cameron et al. 2010) and HAT-P-2 $\left(m_{V}=8.7\right.$, Bakos et al. 2007).

With the launch of the CoRoT (Barge et al. 2008) and Kepler satellites (Borucki et al. 2010) transit searches entered the space era. Without the negative influence of atmospheric scintillation a higher photometric stability can be reached, which allows for the detection of significantly smaller planets. This led to the discovery of the first rocky planets (Léger et al. 2009; Batalha et al. 2011). The NASA Kepler mission stared at a single $115 \mathrm{deg}^{2}$ field for four years, monitoring 150000 stars and finding thousands of transiting planets. This greatly expanded our knowledge of the exoplanet population, almost down to Earth-size planets at Earth-size orbits. Unfortunately, most of the planet host stars are too faint to even obtain the masses of the exoplanets through radial velocity measurements, and detailed atmospheric characterization such as for HD 209458b and HD 189733b is currently not feasible even with the largest telescopes. The more recent K2 mission, utilizing the Kepler satellite after two reaction wheels had failed, does concentrate on brighter stars. However, these are still significantly fainter than the brightest transiting systems expected to exist in the sky at $m_{V}<8$. Even the long-running ground-based SuperWASP and HATNet surveys hardly reach these bright stars. Conversely, radial velocity 


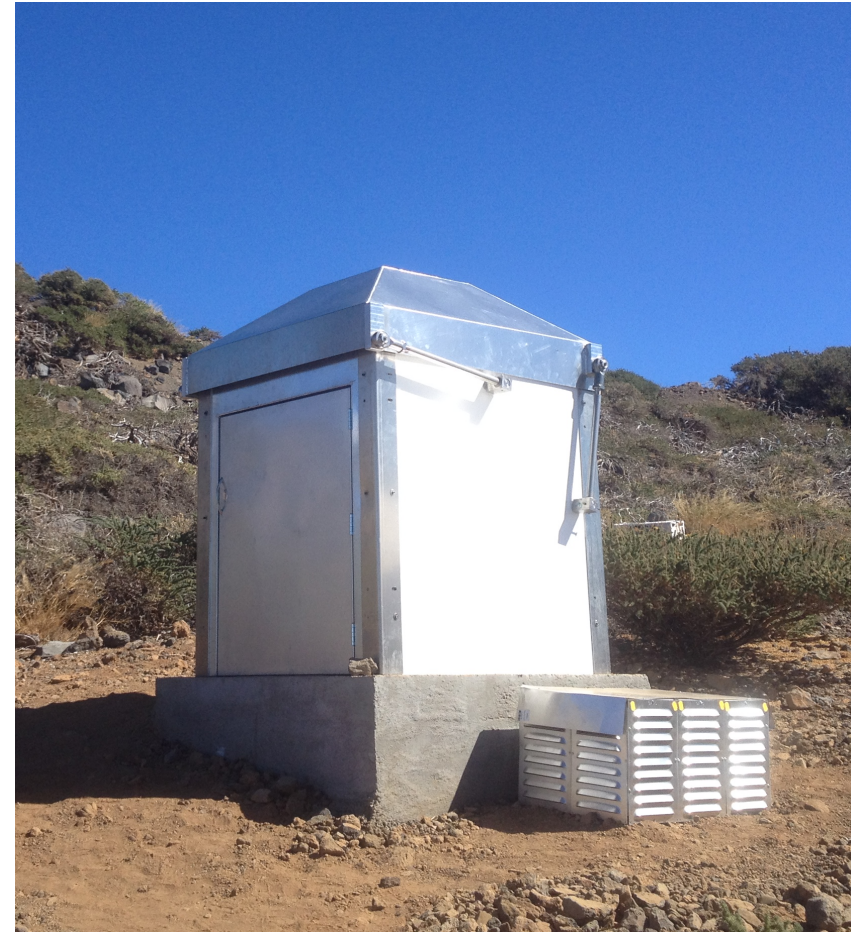

Fig. 1. Northern MASCARA station on La Palma (Spain). The box next to the station contains the heat exchanger used in the decommissioned liquid cooling system.

searches specifically target bright stars, but are limited by the amount of observing time needed for a single star and as such cannot monitor all bright stars. Of the roughly 20000 bright stars with radii $<2 R_{\odot}$ only a few thousand have been searched for planets by means of radial velocity measurements.

For this reason we initiated a new ground-based transit survey project aimed specifically at bright stars, the Multi-Site AllSky CAmeRA ${ }^{1}$ (MASCARA). This paper describes the design and operations of the MASCARA stations in Sects. 2 and 3. The on-site photometry pipeline is outlined in Sect. 4 and the performance of the northern station is presented in Sect. 5. Finally, the place of MASCARA as part of the next generation of transit surveys is discussed in Sect. 6.

\section{Station design}

The brightest stars to exhibit planetary transits are expected at $m_{V}>4$. Transit surveys such as HATNET and SuperWASP cover stars fainter than $m_{V} \sim 8$. This means that the bright end of the transiting exoplanet population was not covered by existing transit surveys. For this reason, stars in the $4<m_{V}<8$ magnitude range form the main target population of MASCARA. Therefore, it is required that $m_{V}=4$ stars do not saturate. Furthermore, over the target magnitude range a precision of at least $1 \%$ per hour should be reached for MASCARA to be sensitive to hot Jupiters transiting solar-type stars. A genuine all-sky survey requires at least one station in both the northern and southern hemisphere to have access to the full sky. Furthermore, the design should be such that a station can be run as robotically as possible with low maintenance for a life time of several years.

A MASCARA station is roughly $1 \times 1 \times 1.5$ metres in size, houses five cameras in a temperature controlled camera

\footnotetext{
1 http://mascara1.strw. leidenuniv.nl/
}

box, and is designed to have a minimum of moving components (the roof and cooling fans). The northern station, shown in Fig. 1, is located at the Observatorio del Roque de los Muchachos $\left(28^{\circ} 45^{\prime} 25^{\prime \prime} \mathrm{N}, 7^{\circ} 53^{\prime} 33^{\prime \prime} \mathrm{E}, 2396 \mathrm{~m}\right)$ on the island of $\mathrm{La}$ Palma (Canary Islands, Spain). The southern station is located at ESO's La Silla Observatory $\left(29^{\circ} 15^{\prime} 40^{\prime \prime} \mathrm{S}, 70^{\circ} 43^{\prime} 53^{\prime \prime} \mathrm{W}, 2400 \mathrm{~m}\right)$ in Chile.

\subsection{Enclosure}

The cameras and electronics are protected by a custom enclosure designed at Leiden Observatory and built by FMI Manufacturing $^{2}$ and the NOVA optical IR group at ASTRON ${ }^{3}$. The enclosure consists of an outer wall with a door that provides access to the electronics inside the station, and a moveable roof. The roof was designed to move to the side of the enclosure to provide access to the entire visible sky. It uses two Kinematics ${ }^{4}$ slewing drives and two motors that can open or close the roof in approximately twenty seconds. In case a single motor fails the other motor is powerful enough to close the roof.

An inner frame inside the enclosure provides the mount for the camera box. The inner frame is mechanically decoupled from the outer structure to minimize variations in the camera pointings that are caused by external forces such as the wind and the motion of the roof. Also attached to the inner frame is an electronics cabinet that contains the control hardware that allows remote operation of the station.

\subsection{Camera box}

The cameras are located inside a camera box with a circular window for each camera. Around each window, four white LEDs with dispersers provide a light source for taking dome flats. Initially, the camera box of the northern station was temperaturecontrolled by means of liquid-cooled Peltier elements and a heat exchanger (see Fig. 1). However, in March 2015, the heat exchanger failed and we have operated without temperaturecontrol for the camera box ever since, though the cameras themselves are still cooled. To be more robust against failures of individual components, the design of the southern station was changed. Excess heat is shifted from the camera box to the enclosure using Peltier elements equipped with heat sinks and subsequently removed from the enclosure by means of eight fans mounted in the outer walls of the enclosure.

Figure 2 shows the sky coverage for both stations. Each station monitors the entire visible sky down to airmass 2 and provides partial coverage down to airmass 3 . For the northern station, the camera pointings correspond to the cardinal directions and zenith. The southern station was rotated $\sim 10^{\circ}$ eastwards to minimize obscuration by the MarLy telescope, and the altitude of the zenith camera was changed from $90^{\circ}$ to $87^{\circ}$ to break a degeneracy in the astrometric solution.

\subsection{Optics}

Figure 3 shows the optical assembly of the northern station mounted inside the camera box. The northern station uses Atik ${ }^{5}$ $11000 \mathrm{M}$ interline CCD cameras that were modified by the manufacturer to allow fast and continuous readout. The southern

\footnotetext{
2 http://www. fmi.nl/

http://www.astron.nl/

4 http://www. kinematicsmfg.com/

5 http://www.atik-cameras.com/
} 

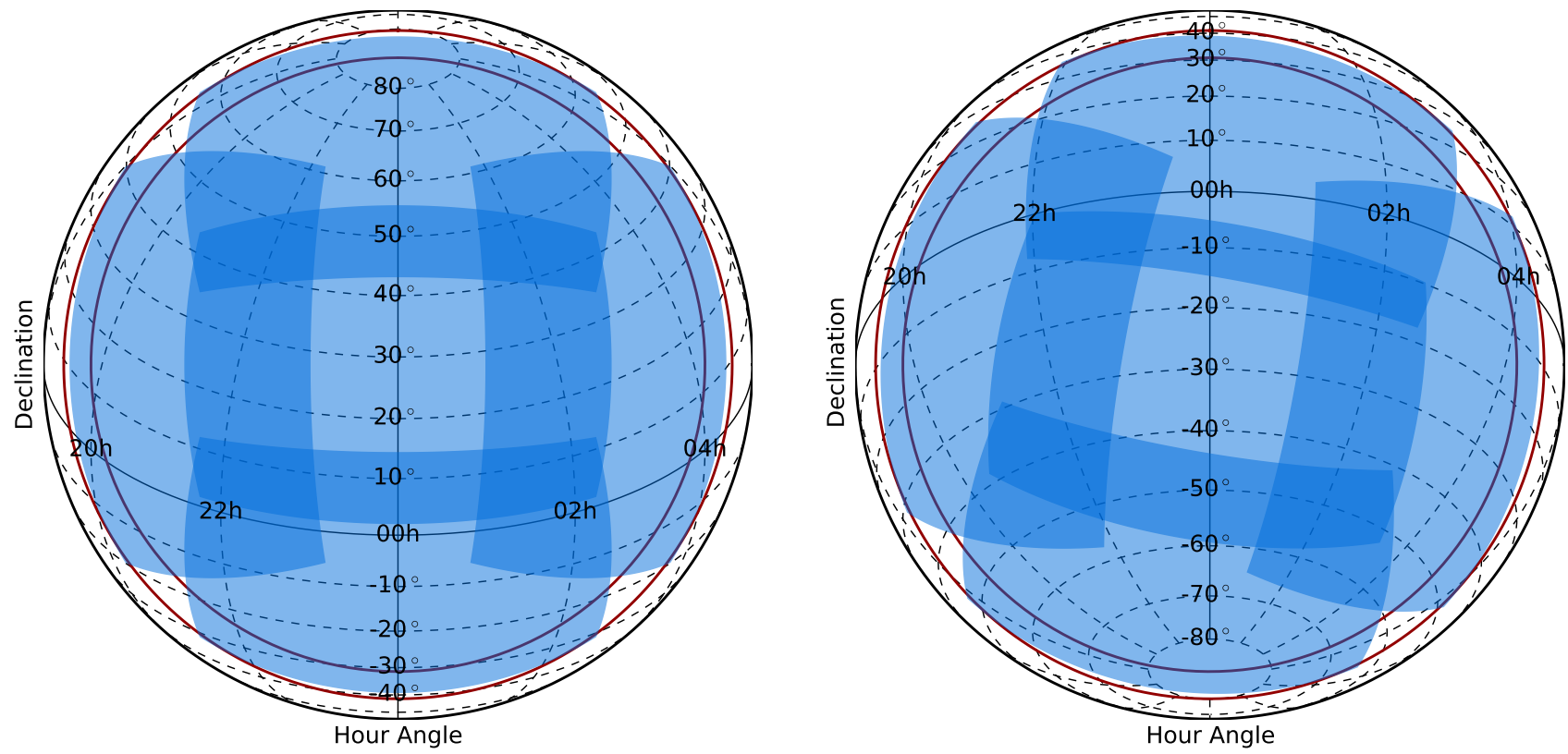

Fig. 2. Field-of-view for the northern (left) and southern (right) station. The coverage of the individual CCDs are indicated in blue. The red lines indicate airmass 2 and 3. The southern station was rotated $\sim 10^{\circ}$ eastwards to minimize obscuration by the MarLy telescope.

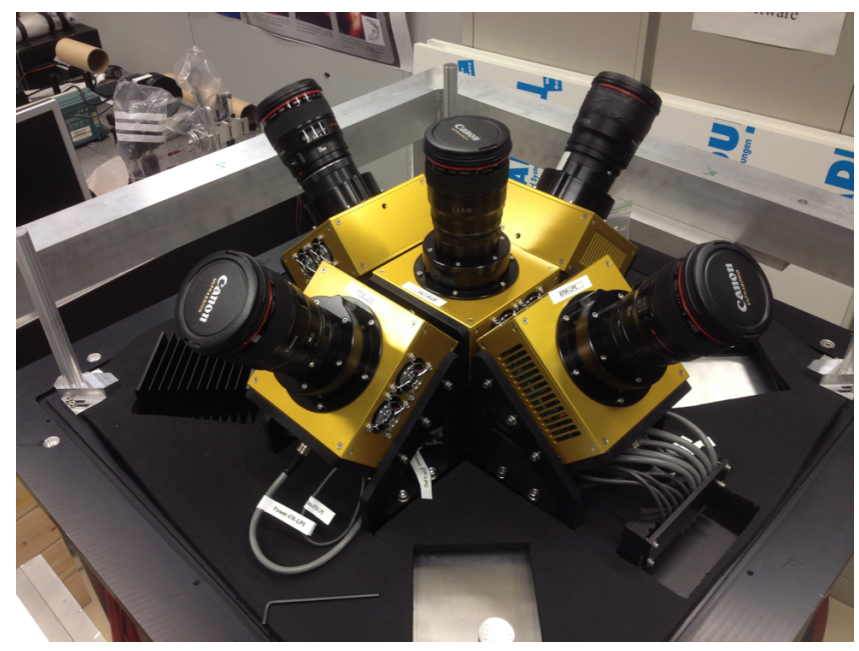

Fig. 3. Atik cameras and $24 \mathrm{~mm}$ lenses of the northern station mounted on the base of the camera box during testing at Leiden Observatory.

station utilizes $\mathrm{FLI}^{6}$ ML11002 interline CCD cameras. Both the Atik and FLI cameras use the Kodak KAI-11002 frontilluminated interline CCD with microlens array. We use interline CCDs because they provide electronic shutters. Each camera takes over 150000 exposures per month prohibiting the use of consumer cameras using shutters, and the use of a separate shutter was deemed to result in undesirable mechanical complexity. Interline CCDs also allow the simultaneous exposing and reading of images, hence eliminating overheads.

The CCDs have $4008 \times 2672$ pixels of $9 \mu \mathrm{m}$, with a peak quantum efficiency of $50 \%$ at $500 \mathrm{~nm}$ (See Fig. 4). The full well depth of the CCD is $60000 \mathrm{e}^{-}$with a $>1000 \times$ anti-blooming protection, which is important when the Moon is in the field. The Atik cameras are read with 16-bit resolution at $12 \mathrm{MHz}$ and have a gain of $0.6 \mathrm{e}^{-} \mathrm{ADU}^{-1}$. We measure the Atik dark

\footnotetext{
6 http://www.flicamera.com/
}

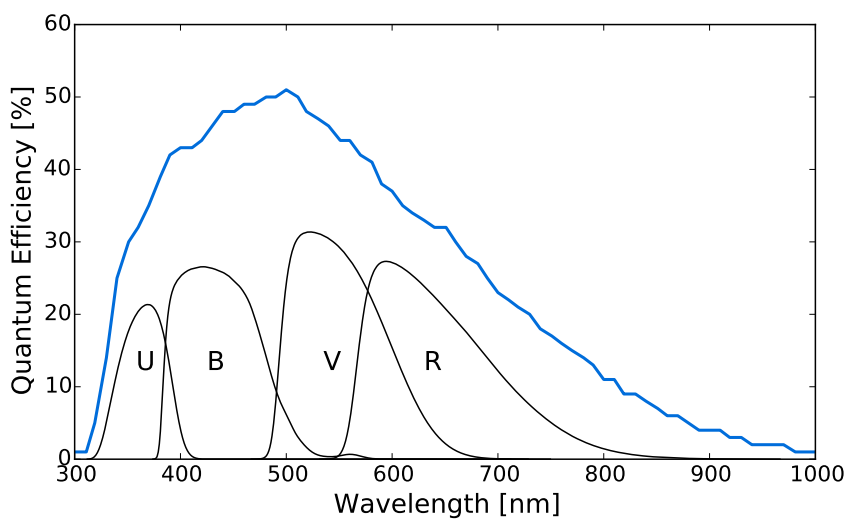

Fig. 4. Quantum efficiency as a function of wavelength for the Kodak KAI-11002 CCD, as provided by the manufacturer. The peak quantum efficiency is $50 \%$ at $500 \mathrm{~nm}$. The $U, B, V$ and $R$ filters are indicated on an arbitrary scale for comparison, and no filter is used in the MASCARA optics.

current and read noise in continuous exposure mode at $-15{ }^{\circ} \mathrm{C}$ to be $\sim 5 \mathrm{ADU} \mathrm{s}^{-1}$ and $25 \mathrm{ADU}$ respectively. The FLI cameras are read with 16-bit resolution at $12 \mathrm{MHz}$, and have a gain of $0.8 \mathrm{e}^{-} \mathrm{ADU}^{-1}$. We measure the FLI dark current and read noise in non-continuous exposure mode at $-10{ }^{\circ} \mathrm{C}$ to be $\sim 0.05 \mathrm{ADU} \mathrm{s}^{-1}$ and $18 \mathrm{ADU}$ respectively. The Atik cameras provide cooling to a maximum of $38^{\circ} \mathrm{C}$ below ambient using a two-stage Peltier and a fan. The FLI cameras provide thermoelectric cooling to a maximum of $55^{\circ} \mathrm{C}$ below ambient.

Each camera is equipped with a Canon $24 \mathrm{~mm} f / 1.4$ USM L II lens with a $17 \mathrm{~mm}$ aperture. These lenses provide a fieldof-view $(\mathrm{FoV})$ of $53^{\circ} \times 74^{\circ}$ per camera and an image scale of $\sim 1^{\prime}$ pixel $^{-1}$. They are slightly defocused to prevent saturation of the $m_{V}=4$ stars and reduce the impact of pixel-to-pixel sensitivity variations. The cameras are not equipped with any filters. 


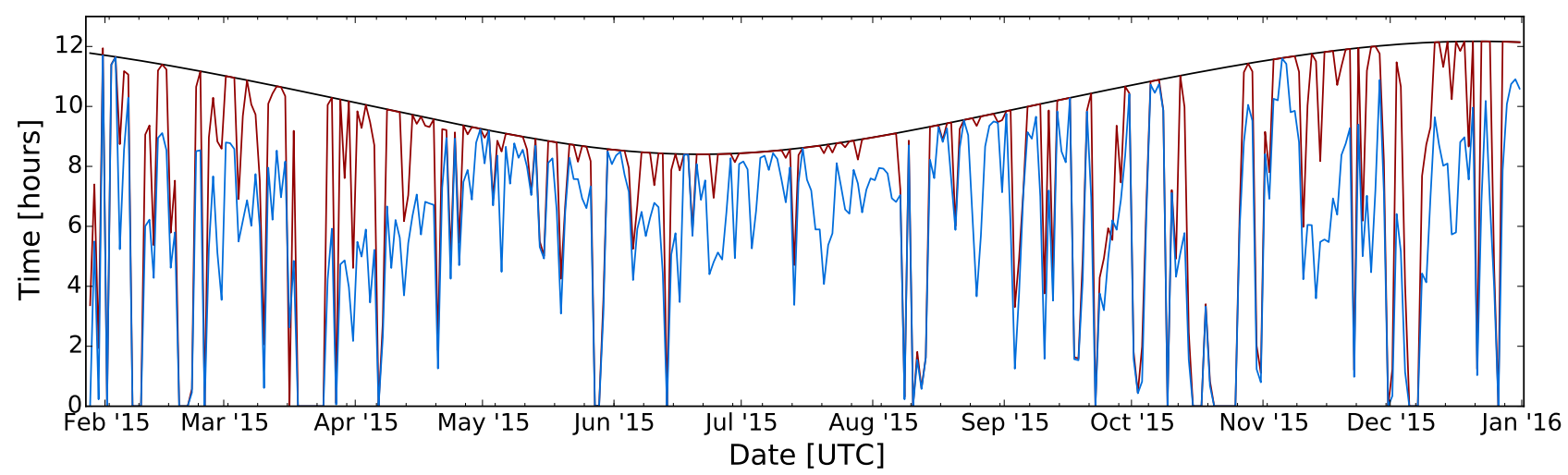

Fig. 5. Observing statistics of 2015 for the northern station. The black line shows the duration of the night, the red line the amount of time the dome was open (i.e. the weather conditions were met), and the blue line the time data was taken averaged over the five cameras.

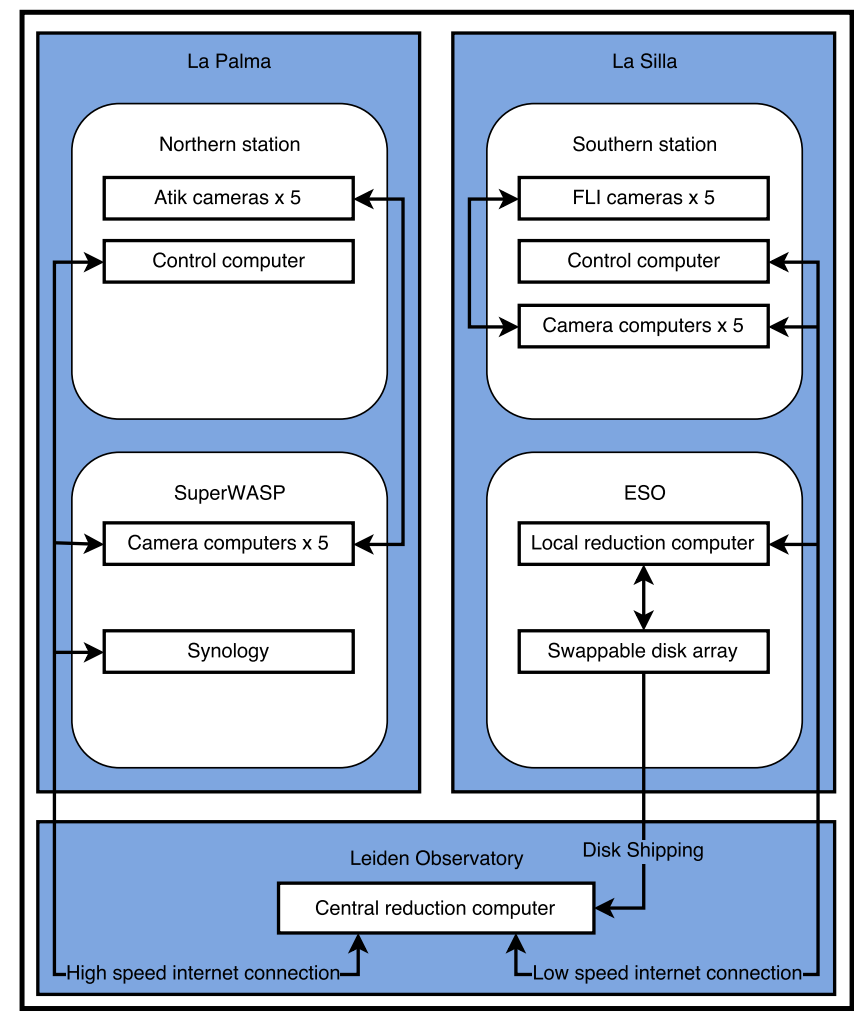

Fig. 6. Schematic representation of the MASCARA project, showing the cameras and computers at the northern and southern station, and the data centre.

\subsection{Electronics and computing}

Figure 6 shows a schematic overview of the hardware used at the northern and southern stations and the data centre at Leiden Observatory. Each station is operated by six computers, comprising one control computer and five camera computers. A GPS clock is used for accurate time-keeping and three sensors monitor the temperature and humidity in the enclosure, camera box and electronics cabinet. Two uninterruptible power supplies (UPSs) channel power to the station.

The northern station is operated in collaboration with SuperWASP (Pollacco et al. 2006). The control computer and Globalsat BU353S4 GPS clock are located inside the MASCARA enclosure, whereas the camera computers, data storage, and UPSs are positioned inside the SuperWASP enclosure. For the first eighteen months of operations the camera computers were connected to the cameras through 20-m USB cables, but these were replaced by USB optical fibres in August 2016 after being identified as a source of unreliability. All computers are HP ProDesk 490 G1 computers running Windows 8 , containing a 500 GB SSD and a 4 TB HDD. Additional data storage and backup is provided by a Synology DS1813+ data storage system operating in RAID-6, resulting in $22 \mathrm{~TB}$ of effective disk space, which is enough for up to four weeks of raw and reduced data. The light curves produced by the northern station are transferred to the data centre at Leiden Observatory on daily basis through a high speed internet connection. Weather information is obtained from the SuperWASP weather station.

All control and camera computers of the southern station are located inside the MASCARA enclosure. Rack-mounted Dell Poweredge R230 E3-1240 v5 computers are used running Windows 10. Each computer contains a $1 \mathrm{~TB}$ SSD and an 8 TB HDD. For time-keeping, a Galleon NTS-4000-GPS-R GPS server is used. An optical fibre connects the station to the local ESO server room where the local reduction computer, a Dell Poweredge R730xd 2x Xeon E5-2660 v3, provides 64 TB of effective storage on a RAID-6 system, which is enough for three months of raw and reduced data. The light curves produced by the southern station are transferred to the data centre at Leiden Observatory by means of disk shipping every three months, using two 8 TB swappable disks. To optimize our work-flow and spot potential problems with the data early, additional analysis of the light curves is performed on-site using the local reduction computer. Weather information is obtained from the available La Silla weather stations.

At the MASCARA data centre at Leiden Observatory we have the central reduction computer, a Dell Poweredge R720xd $2 x$ Xeon E5-2660, which is used for all further analysis of the data taken by the MASCARA stations. It currently provides 44 TB of effective disk space on a RAID-6 system, and will be expanded as needed in the future. In addition to the data archive kept on the central reduction computer the data are also backedup to separate disks.

\section{Nominal operations}

Each MASCARA station is fully automated, and is operated through python software running on the control computer. Each of the cameras is controlled by its own camera computer, which is slaved to the control computer. The cameras are cooled to $-15^{\circ} \mathrm{C}$ during the night. Observations are taken in continuous exposure mode at fixed sidereal times with an integration time of 6.4 sidereal seconds. We opted for observations at fixed sidereal 
times to make it possible for difference imaging techniques to be applied to the data, despite the fixed pointings. However, this feature is currently not used in the data analysis. The exposure time was motivated by the saturation level of bright stars, readtime of the detector, and the desire to fit an integer number of exposures in one sidereal day.

Preparations for the night's observations start when the sun altitude passes $0^{\circ}$. Each camera starts by taking a series of calibration frames consisting of twenty bias frames, dark frames and dome flats. The dome flats are only taken for instrumental monitoring purposes and are not used for the data analysis. The darks and flats are taken in continuous exposure mode with the same $6.4 \mathrm{~s}$ exposure time as the science observations. When the sun altitude passes $-10^{\circ}$ the weather conditions are assessed. The weather is considered suitable for observing if all of the following conditions are met:

- No rain is detected.

- The outside humidity is below $80 \%$.

- The outside temperature exceeds $0{ }^{\circ} \mathrm{C}$.

- The wind speed is below $50 \mathrm{~km} \mathrm{~h}^{-1}$.

- The sky temperature is below $-25^{\circ} \mathrm{C}$, which is indicative of clear skies.

- The humidity inside the enclosure is below $90 \%$.

- The temperature inside the camera box is below $50{ }^{\circ} \mathrm{C}$.

- Communication with the weather station and the humidity sensors is working.

If all conditions are met at the beginning of the night, the dome is opened and science images are taken. If any of the conditions cease to be met the dome is closed and is not re-opened until all conditions are met again for fifteen consecutive minutes. The observations end when the sun altitude again passes $-10^{\circ}$. At this time the dome is closed and a second series of calibration frames is taken.

During the day, an on-site reduction pipeline is run on the data taken during the preceding night. The data products of this pipeline are transferred to Leiden Observatory whereas the raw data is backed-up on the HDD drives of the camera computers and (for the northern station) the Synology storage system. Sufficient storage space is present to archive at least four weeks of raw data before deletion. In principle, this will allow for the search for transient events in the raw data.

\section{Data analysis}

The MASCARA data analysis pipeline consists of two consecutive parts: the on-site reduction pipeline discussed here, and the post-processing pipeline which will be described in an forthcoming paper.

\subsection{On-site reduction pipeline}

Because each of the five cameras takes one $22 \mathrm{MB}$ image every $6.4 \mathrm{~s}$, a single station produces up to $600 \mathrm{~GB}$ of raw data per night. Neither the transfer nor the long-term saving of the raw data is feasible for budgetary reasons. Instead, an on-site reduction pipeline performs image calibration, astrometry and photometry, producing light curves and binned images which amount to $\sim 25 \mathrm{~GB}$ per night per station. For the northern station these data products are transferred to Leiden data centre via the internet. For the southern station the data are shipped to Leiden on disk every three months.

\subsubsection{Calibration and astrometry}

Biases, dark frames, and dome flats taken at the beginning of the night are processed to produce a master dark and master flat, as is done for the calibration frames taken at the end of the night. The mean $\mu_{\mathrm{b}}$ and rms $\sigma_{\mathrm{b}}$ of the master bias is used to asses the quality of the darks. Any dark with a mean, $\mu_{\mathrm{d}}$, exceeding $\mu_{\mathrm{b}}+2 \sigma_{\mathrm{b}}$ is rejected and the remaining darks are averaged together to create the master dark. If there are fewer than ten good darks, no master dark is created and the last master dark from a previous night is used instead. Although a master flat is created, it is not used in our analysis because we found that including the flatfield correction reduced the quality of the photometry. We believe this reduction in photometric quality is a result of large scale non-uniformity in the illumination used, resulting in insufficient stability in the flatfield images. The master dark is subtracted from each science frame to correct for the bias and darkcurrent of the camera, as well as providing a first-order correction for hot pixels.

The pipeline uses the All-Sky Compiled Catalogue (ASCC, Kharchenko 2001; Kharchenko \& Roeser 2009) as input for the astrometry and photometry. Stars brighter than $m_{V}=7$, which typically number 1500 stars per image, are used to find the astrometric solution. The solution consists of the standard world coordinate system (WCS) transformations and two distortion polynomials. The distortion polynomials are necessary to correct for a predominantly radial offset between the WCS positions and the observed positions. We use sixth-order polynomials in the WCS positions without higher-order cross-terms to fit the residuals between the observed and the WCS-only positions.

The astrometric solution is updated at the beginning of the night, at every fiftieth image in the exposure sequence and after interruptions in the data taking. The parameters of the WCS transformations are only updated when the astrometric solution has changed by more than 2 pixels, otherwise only the distortion polynomials are updated.

\subsubsection{Binned images}

Every sequence of fifty images is stacked to create a binned image. Because the cameras have a fixed pointing, significant shifts need to be applied to the images to correct for the motion of the stars. In addition, the large FoV means the magnitude and direction of the shifts vary across the image. Therefore each image is divided into tiles of $32 \times 32$ pixels, discarding four rows at the top and bottom of the image and eight columns on both the left and right. The centre of each of these tiles is subsequently shifted to its position at the mid-time of the fifty images, using only the WCS transformations. Therefore, tiles are shifted in time by a maximum of $160 \mathrm{~s}$ and the maximum offset on the position of the pixels near the edge of a tile is 0.3 pixels. Tiles are then added to the stack using nearest neighbour interpolation, introducing a maximum offset of 0.7 pixels. When the last image has been added to the stack, it is divided by the total number of individual images added at each pixel to produce the binned image. The binned images are saved using the Hcompress ${ }^{7}$ algorithm using a scale parameter of one, resulting in file sizes of $5 \mathrm{MB}$ that then total $\sim 0.5 \mathrm{~GB}$ per camera per night. The binned images allow us to store all of the collected data at reduced cadence and make it possible to study fainter stars.

7 http://www.stsci.edu/software/hcompress.html 


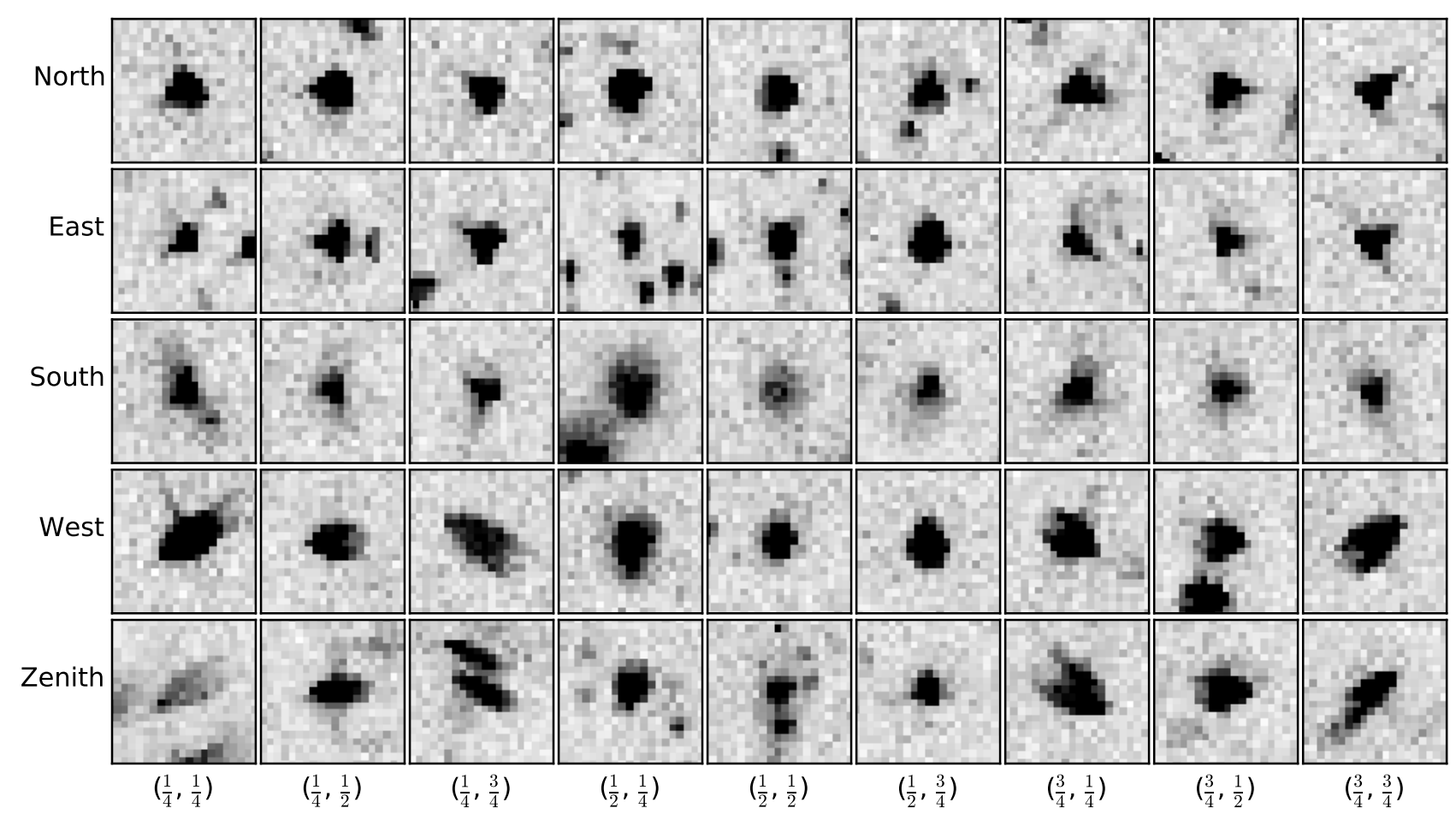

Fig. 7. Representative PSFs for the cameras of the northern station, taken from the 6.4 s exposures made by the cameras on July $7201623: 42: 16$ UT. Stars with $6<m_{V}<7$ were selected close to the points of a $3 \times 3$ grid across the CCD with coordinates at $\frac{1}{4}, \frac{1}{2}$ and $\frac{3}{4}$ the size of the image in both $x$ and $y$. The colour scale of each PSF was adjusted to run from -3 to 9 times the standard deviation on the local background. Near the centre (column $\left(\frac{1}{2}, \frac{1}{2}\right)$ ) the PSFs are symmetric, whereas toward the edges they are distorted by varying degrees of coma that are introduced by the optics.

\subsubsection{Photometry}

Aperture photometry is performed on each individual image for stars with $2<m_{V}<8.4$ in the ASCC catalogue, which typically number 6500 stars per image. The CCD coordinates of the stars are obtained using the astrometric solution, and aperture photometry is performed in apertures of 2.5 and 4.5 pixels. The sky background is estimated in an annulus between 6 and 21 pixels. We refer to this photometry as the "fast" light curves. "Slow" light curves are similarly obtained by performing aperture photometry on the binned images for stars down to $m_{V}<10$, which typically number 40000 stars per image. Photometry on the binned images is performed before compression. The astrometric corrections are re-evaluated on the binned image before computing the star positions. For the slow light curves, photometry is performed in apertures of $2.5,3.5,4.5$, and 5.5 pixels, and the sky background is estimated in an annulus between 6 and 21 pixels. The $6.4 \mathrm{~s}$ fast cadence and $320 \mathrm{~s}$ slow cadence light curves are saved in the HDF5 file format resulting in file sizes of $\sim 3 \mathrm{~GB}$ and $\sim 0.5 \mathrm{~GB}$ per camera per night.

The on-site reduction pipeline takes between $6-10 \mathrm{~h}$ to complete for a full night of data. Figures showing the reduced light curves of several bright stars are automatically produced each night for each camera and sent to the MASCARA team to serve as a daily monitor of the MASCARA system.

\section{Performance}

The northern station was commisioned on La Palma in October 2014. The southern station will be commissioned in early 2017. In this section, we discuss the performance and data quality of the northern station using the first year of data.

\subsection{Station performance}

After one month of operation, the northern station experienced an increasing number of camera failures. Subsequently, observations ceased completely at the end of November 2014. The cause of the camera failures was identified as submersion of the amplifier units on the 20-m USB cables in the cable duct between the MASCARA station and the SuperWASP enclosure. The USB cables were replaced late January 2015, making sure the amplifiers were located inside the SuperWASP enclosure. Figure 5 presents the observing statistics for the year 2015, showing the number of dark hours, hours of good weather, and hours of data taken per night. On average, about $22 \%$ of good weather was lost to camera failures, mostly in winter. We carefully monitored these failures and identified humidity, cross-talk between the cables, and grounding as possible causes for the failures. In August 2016, we replaced the USB cables again, this time with USB optical fibres, and from that moment we have had nearly perfect (99\%) uptime on the cameras.

In March 2015, temperature-control of the camera box was lost when the heat exchanger failed. Because the cameras themselves have limited ability to cool relative to the camera box ambient temperature $T_{\mathrm{cb}}$, we had to change the temperature of the cameras to be $-15{ }^{\circ} \mathrm{C}$ when possible, and $T_{\mathrm{cb}}-40{ }^{\circ} \mathrm{C}$ otherwise. In July 2015, an on-site inspection of the heat exchanger found that the failure had most likely been caused by a dust storm from the Sahara desert (Calima). Because it was probable that a new heat exchanger would break down in the same fashion, a decision was made not to replace the heat exchanger. Despite a slight increase in thermal noise, the impact of losing the heat exchanger has been minimal and we still easily achieve our original target precision of $1 \%$ per hour. For the southern station, we changed the design to cool the camera box by means of aircooled Peltiers. 


\section{G. J. J. Talens et al.: The Multi-site All-Sky CAmeRA (MASCARA)}
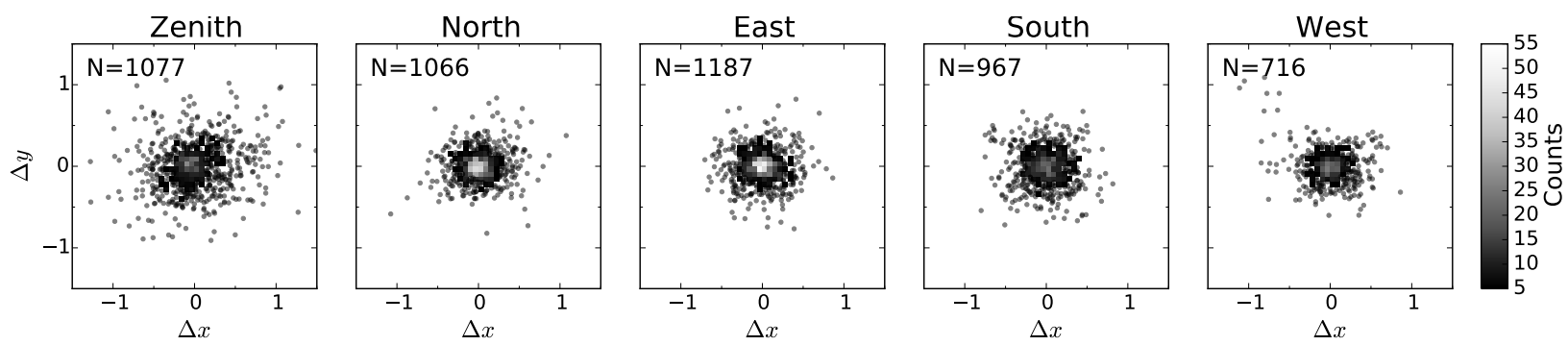

Fig. 8. Residuals between the observed and predicted stellar position for the cameras of the northern station for an image taken on July 112016 00:05:08 UT. Each panel shows the difference in the observed and predicted $x, y$ positions for approximately 1000 stars used in the astrometric solution. Typical standard deviations in both the $x$ and $y$ residuals are $\sim 0.2$ pixels for the north, east, south and west cameras and $\sim 0.3$ pixels for the zenith camera, probably due to stronger vignetting.
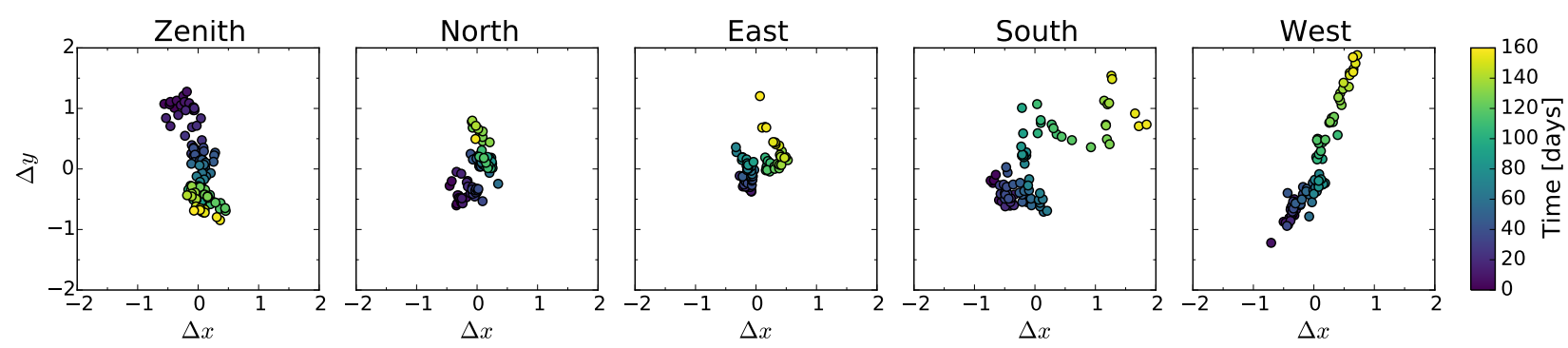

Fig. 9. Change in the pointing of the cameras of the northern station during a 160 day period between July 162015 and December 232015 . Each panel shows the mean subtracted $x, y$ position of a particular star at a fixed sidereal time, chosen such that its location is close to the centre of the CCD. The panels show drifts in the pointing of the individual cameras of 1-2 pixels over this five months period.

\subsection{The point spread function and astrometry}

Figure 7 shows the point spread function (PSF) of stars with $6<$ $m_{V}<7$ on a $3 \times 3$ grid across the CCDs of the northern station. The PSFs show the effects of the slight defocus and aberrations inherent to wide field optical systems, most importantly coma. Stars in the centre of the CCD have symmetric PSFs whereas those in the corners are strongly distorted.

Figure 8 shows the residuals between the detected stellar positions and the positions predicted by the astrometric solution for images taken on July 11 2016 00:05:08 UT. The residuals show a symmetric distribution around zero with typical standard deviation of $\sim 0.2$ pixels in both $x$ and $y$ for the north, east, south and west cameras. The standard deviation on the residuals of the zenith camera is slightly larger at $\sim 0.3$ pixels, which is likely caused by stronger vignetting in this camera resulting in larger uncertainties in the measured stellar positions. Figure 9 shows the change in the $x, y$ position of a fixed point on the sky at a fixed sidereal time over an observing season. In the ideal case, when the MASCARA cameras undergo no changes in pointing, the $x, y$ positions stay constant at the origin. It can be seen that the pointing of the cameras changes by only a few pixels over the 160 day period shown. These are most likely caused by thermal expansion and contraction of components such as the inner frame and the lens mounts. Overall, the pointing stability of the cameras are of the order of 1-2 pixels over long timescales. Not shown in the figure are the typical nightly variations in the pointing that are caused by thermal settling of the lens-camera system, which is typically of the order of a few tenths of pixels.

\subsection{Photometry}

Figure 10 shows an example of a raw light curve taken by the northern station. Two kinds of spatial systematics are evident in the light curve, vignetting and intrapixel variations. The vignetting is responsible for the overall shape of the light curve and is the result of a decrease in the transmission of the optics for off-axis light rays found in many wide-field optical systems. Intrapixel variations, suspected to be mainly due to the microlens array blocking a changing fraction of the total light as the star moves across the CCD, are responsible for sinusoidal modulations seen most clearly in the zenith camera. Not directly visible in this light curve are the effects of temporal systematics (e.g. atmospheric transmission) and PSF variations, the latter of which modifies the fraction of the total starlight inside the photometric aperture.

A detailed description of the analysis required to remove these systematic effects is beyond the scope of this text, but will be given in a forthcoming paper. Here we briefly outline the analysis so we can discuss the quality of the final light curves. In short, we employ a version of the coarse decorrelation algorithm (Collier Cameron et al. 2006) that was altered to include corrections for MASCARA's spatial systematics in order to remove the spatial and temporal systematics present in the data. Next, the data are binned by 50 points to a cadence of $320 \mathrm{~s}$. Finally, the PSF variations are corrected by fitting sinusoids in the local sidereal time to the binned data for each individual star.

Figure 11 shows the root mean square (rms) scatter on the final binned light curves for the west camera of the northern station. We achieve an RMS of $\sim 2 \%$ at $m_{V}=8.4$ going down to $\sim 0.5 \%$ at $m_{V}=4$, and similar results for the other cameras. At the faint end performance is limited by the photon noise of the sky background, whereas at the bright end the limiting factors are scintillation noise and residual systematics.

\section{Discussion and conclusion}

In the magnitude range targeted by MASCARA we expect to find a few (2-5) hot Jupiters around Sun-like (FGK) stars and a similar number around earlier spectral types (Snellen et al. 2012). To show that MASCARA is indeed capable of finding these sorts of systems, as well as demonstrate its potential 


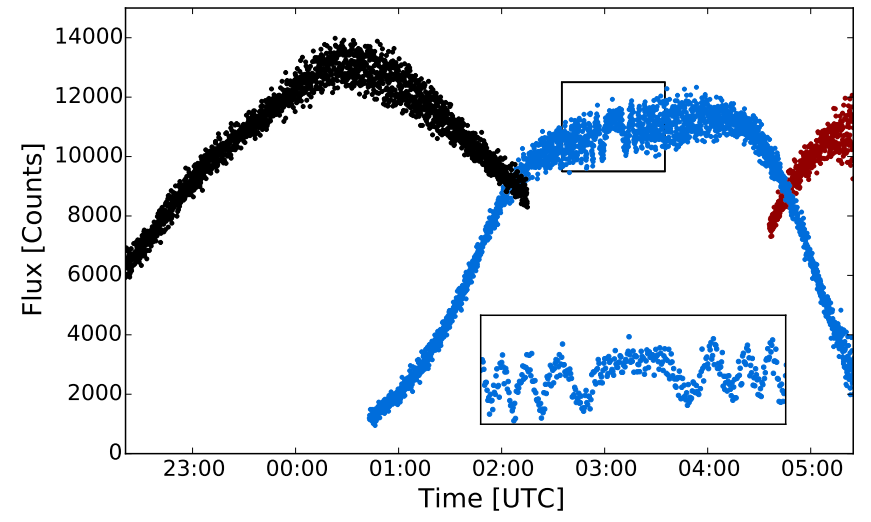

Fig. 10. Raw light curve of HD 189733 taken by the northern station on June 18 2015. The photometry in the 2.5 pixel aperture is shown for the west (red), zenith (blue) and east (black) cameras. The effects of vignetting are visible for all cameras, causing a decrease in flux near the edges of the CCD. The effects of intrapixel variations are most visible in the zenith camera, causing the sinusoidal modulations in the centre of the light curve.

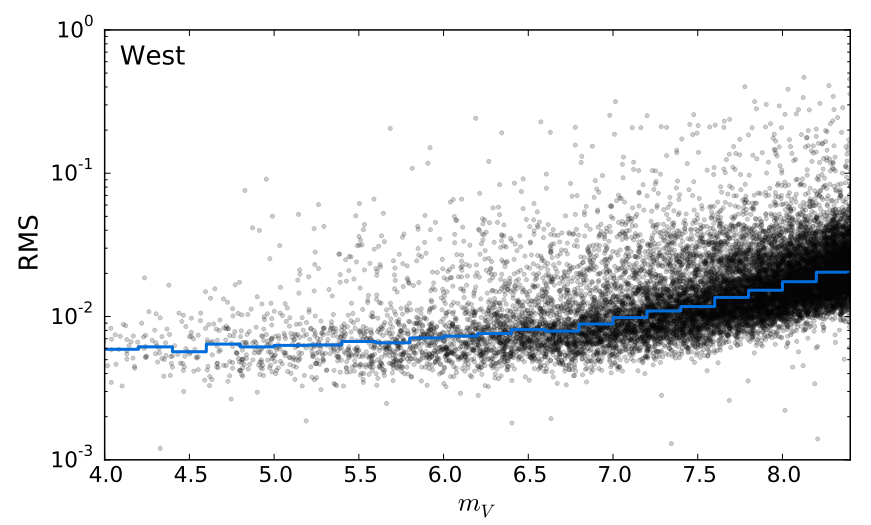

Fig. 11. RMS achieved for the west camera of the northern station during the second quarter of 2015. Shown are the RMS on the five-minute binned light curves of individual stars with $>500$ data points (black points) and the median relation in bins of $\Delta m_{V}=0.2$ (blue line). We achieve an RMS of $\sim 0.5 \%$ at the bright end of our magnitude range and $\sim 2 \%$ at the faint end. Similar RMS is achieved by the other cameras.

for variable star research, Fig. 12 shows the phase-folded light curves for the known transiting exoplanet host HD 189733 and the star RR Lyrae. HD 189733 is a magnitude $m_{V}=7.7$ star orbited by a hot Jupiter with a period of $P=2.21857312$ days and a transit depth of $\sim 2.2 \%$ (Bouchy et al. 2005). The transit is clearly visible in the folded MASCARA data, which have an RMS of $\sim 1 \%$. RR Lyrae is a magnitude $m_{V}=7.1$ star that shows, and gives its name to, a type of periodic variability. For RR Lyrae this variability has a period of $P=0.56677439$ days and the data have been folded to twice this period.

A number of surveys with a similar all-sky design, but targeting fainter stars, are in various stages of development and operation, for example HATPI ${ }^{8}$, Evryscope (Law et al. 2015) and the Fly's Eye Camera System (Pál et al. 2013). These surveys employ a similar strategy of utilizing multiple cameras attached to a single mount to survey large fractions of the visible sky simultaneously, but they employ tracking on this mount as opposed to MASCARA's fixed pointings. Besides MASCARA, several other transit surveys searching for targets suitable for characterization have come online in recent years,

\footnotetext{
8 http://hatpi.org/
}
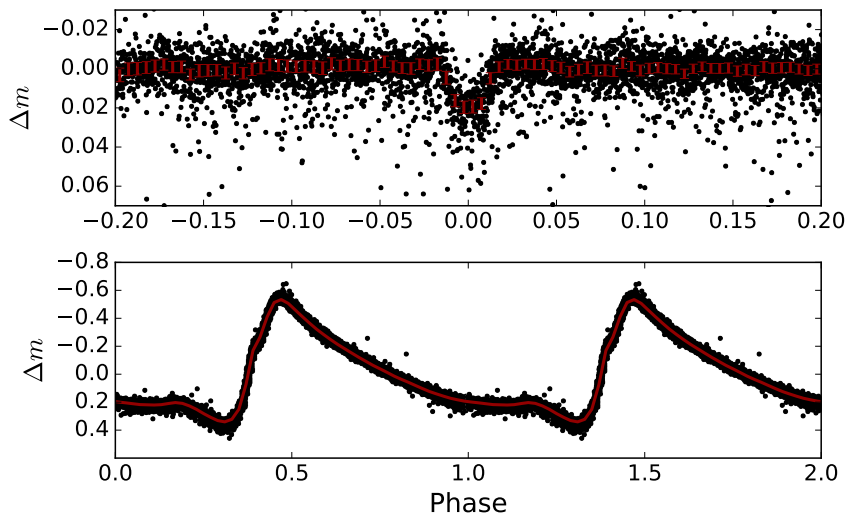

Fig. 12. Light curves of HD 189733 (top) and RR Lyrae (bottom). For HD 189733 the reduced data (black points) have been folded to the orbital period of HD 189733b and the folded light curve has been binned by 0.005 in phase (red errorbars). The errorbars have been scaled by a factor five for clarity. For RR Lyrae the reduced data has been folded to twice the period of its variability (black points) and the folded light curve has been binned by 0.02 in phase (red line). We note that the scatter away from the red line is primarily caused by residual systematics in the data taken by the zenith camera, which suffers from the strongest vignetting.

for example the Next Generation Transit Survey (NGTS), the Kilodegree Extremely Little Telescope (KELT), the TRAnsiting Planets and PlanetesImals Small Telescope (TRAPPIST) and MEarth (West et al. 2016; Pepper et al. 2007; Jehin et al. 2011; Nutzman \& Charbonneau 2008). The NGTS focuses on achieving greater precision on fainter stars $\left(m_{V}<13\right)$ to push towards Neptune- and Earth-sized planets that are suitable for characterization with the James Web Space Telescope (JWST) and the European Extremely Large Telescope (E-ELT). The KELT survey targets stars with magnitudes $8<m_{V}<11$, just below the primary target population of MASCARA. They have recently published the detection of a planet around KELT-11 (Pepper et al. 2017), their brightest confirmed planet host to date at $m_{V}=8.0$. TRAPPIST and MEarth target M-dwarfs in an effort to detect Earth-size planets, resulting in the detection of GJ1214b (Charbonneau et al. 2009), GJ 1132b (Berta-Thompson et al. 2015), and TRAPPIST-1b and c (Gillon et al. 2016). However, MASCARA aims to provide continuous and all-sky coverage of targets in the magnitude range $4<m_{V}<8$, significantly expanding the discovery space towards brighter stars.

During the MASCARA design phase NASA approved the Transiting Exoplanet Survey Satellite (TESS, Ricker et al. 2015), whose all-sky survey will include the same magnitude range as MASCARA. Fortunately, with a TESS launch date of early 2018, MASCARA has a head start of several years. Furthermore, for a large part of the sky TESS will monitor stars for only four weeks. This will allow for an interesting synergy between MASCARA and TESS. Although the photometric precision of MASCARA is between one and two orders of magnitude lower than that achieved by TESS, it will provide light curves of at least several years for all targets, thus providing valuable information on long-term variability and revealing longer period planets in the combined TESS + MASCARA data.

In conclusion, the northern and southern MASCARA stations survey all bright stars, $4<m_{V}<8$, and the northern station has been shown to achieve a precision of $1.5 \%$ per five minutes, well in excess of the original goal of $1 \%$ per hour. In addition to its primary goal of finding transiting exoplanets MASCARA will also create an archive of 70000 light curves suitable for studying 


\section{G. J. J. Talens et al.: The Multi-site All-Sky CAmeRA (MASCARA)}

variable stars. As such, MASCARA is capable of finding nearly any hot Jupiter transiting a solar-type star in the targeted magnitude range, and will provide new targets for exoplanet characterization.

Acknowledgements. We thank the anonymous referee for their helpful comments and suggestions. IS acknowledges support from a NWO VICI grant (639.043.107). This project has received funding from the European Research Council (ERC) under the European Union's Horizon 2020 research and innovation programme (grant agreement no. 694513). We thank Johan Pragt, Sjouke Kuindersma, Menno de Haan and Eddy Elswijk of ASTRON and the NOVA Optical IR group for their aid in production of the enclosure and final testing at Dwingeloo. We thank Alan Chopping and Juerg Rey of the Isaac Newton Group for their support during the commissioning of the northern station. We extend our thanks to the Director and staff of the Isaac Newton Group of Telescopes for their support of the MASCARA operations. We have benefited greatly from the publicly available programming language PYTHON, including the NUMPY, MATPLOTLIB, PYFITS, SCIPY and H5PY packages

\section{References}

Alonso, R., Brown, T. M., Torres, G., et al. 2004, ApJ, 613, L153 Bakos, G., Noyes, R. W., Kovács, G., et al. 2004, PASP, 116, 266

Bakos, G. Á., Kovács, G., Torres, G., et al. 2007, ApJ, 670, 826

Barge, P., Baglin, A., Auvergne, M., et al. 2008, A\&A, 482, L17

Batalha, N. M., Borucki, W. J., Bryson, S. T., et al. 2011, ApJ, 729, 27

Berta-Thompson, Z. K., Irwin, J., Charbonneau, D., et al. 2015, Nature, 527, 204

Borucki, W. J., Caldwell, D., Koch, D. G., et al. 2001, PASP, 113, 439

Borucki, W. J., Koch, D., Basri, G., et al. 2010, Science, 327, 977

Bouchy, F., Udry, S., Mayor, M., et al. 2005, A\&A, 444, L15

Brown, T. M., \& Charbonneau, D. 2000, in Disks, Planetesimals, and Planets, eds. G. Garzón, C. Eiroa, D. de Winter, \& T. J. Mahoney, ASP Conf. Ser. 219,584
Butler, R. P., Marcy, G. W., Williams, E., Hauser, H., \& Shirts, P. 1997, ApJ, 474, L115

Charbonneau, D., Brown, T. M., Latham, D. W., \& Mayor, M. 2000, ApJ, 529, L45

Charbonneau, D., Brown, T. M., Noyes, R. W., \& Gilliland, R. L. 2002, ApJ, 568,377

Charbonneau, D., Berta, Z. K., Irwin, J., et al. 2009, Nature, 462, 891

Collier Cameron, A., Pollacco, D., Street, R. A., et al. 2006, MNRAS, 373 799

Collier Cameron, A., Guenther, E., Smalley, B., et al. 2010, MNRAS, 407, 507

Gillon, M., Jehin, E., Lederer, S. M., et al. 2016, Nature, 533, 221

Henry, G. W., Marcy, G. W., Butler, R. P., \& Vogt, S. S. 2000, ApJ, 529, L41

Horne, K. 2001, in Techniques for the Detection of Planets and Life beyond the Solar System, ed. W. R. F. Dent, 5

Jehin, E., Gillon, M., Queloz, D., et al. 2011, The Messenger, 145, 2

Kharchenko, N. V. 2001, Kinematika i Fizika Nebesnykh Tel, 17, 409

Kharchenko, N. V., \& Roeser, S. 2009, VizieR Online Data Catalog: I/280

Konacki, M., Torres, G., Jha, S., \& Sasselov, D. D. 2003, Nature, 421, 507

Law, N. M., Fors, O., Ratzloff, J., et al. 2015, PASP, 127, 234

Léger, A., Rouan, D., Schneider, J., et al. 2009, A\&A, 506, 287

Mayor, M., \& Queloz, D. 1995, Nature, 378, 355

McCullough, P. R., Stys, J. E., Valenti, J. A., et al. 2005, PASP, 117, 783

Nutzman, P., \& Charbonneau, D. 2008, PASP, 120, 317

Pál, A., Mészáros, L., Csépány, G., et al. 2013, Astron. Nachr., 334, 932

Pepper, J., Pogge, R. W., DePoy, D. L., et al. 2007, PASP, 119, 923

Pepper, J., Rodriguez, J. E., Collins, K. A., et al. 2017, AAS, submitted [arXiv: 1607.01755]

Pollacco, D. L., Skillen, I., Collier Cameron, A., et al. 2006, PASP, 118, 1407

Ricker, G. R., Winn, J. N., Vanderspek, R., et al. 2015, J. Astron. Telescopes, Instruments, and Systems, 1, 014003

Snellen, I. A. G., Stuik, R., Navarro, R., et al. 2012, in Ground-based and Airborne Telescopes IV, Proc. SPIE, 8444, 84440I

West, R. G., Pollacco, D., Wheatley, P., et al. 2016, The Messenger, 165, 10 